\title{
Deafferentation Effects in Lateral Cuneate Nucleus of the Cat: Correlation of Structural Alterations with Firing Pattern Changes
}

\author{
Terrell D. Kjerulf, John T. O’Neal, William H. Calvin, \\ John D. Loeser and Lesnick E. Westrum ${ }^{1}$ \\ Department of Neurological Surgery, University of Washington School of \\ Medicine, Seattle, Washington 98195
}

Received September 23, 1972

A correlated anatomical and physiological investigation of the effects of unilateral cervicothoracic dorsal rhizotomies upon lateral cuneate nucleus of the cat ( $\mathrm{LCN}$ ) is reported. Pairs of adult cats with identical survival times were selected to correlate structural and functional changes. Two phases are described in the development of alterations of neuronal firing patterns. In the first phase, a relative silence within LCN was associated with depletion of round synaptic vesicles in the presynaptic profiles (LR boutons) of primary dorsal root afferents. The second phase was characterized by a development of spontaneous electrical hyperactivity which corresponded anatomically to the presence of denuded postsynaptic specializations, transient increase of adjacent extracellular space and an apparent decrease in the number of dendritic spines. There was a persistence of an unaltered population of small presynaptic boutons with flattened vesicles (SF boutons). The LCN neuronal membrane is viewed as having an intrinsic tendency for repetitive firing which is enhanced by the functional effects of denuded postsynaptic specialization. A marked similarity was found between some of the spontaneous firing patterns of normal animals (doublets) and the high frequency bursting firing pattern in deafferented preparation. Three models for repetitive spike production are considered in our analysis: oscillator-produced spikes; EPSP-produced spikes; and spike-evoked spikes. The spike-evoked spikes model is considered to be the

1 The authors thank William Congdon, Jerrold Maddocks, Vladimir Uhlir and Patricia Yadock for their assistance in these experiments. This work was supported in part by Grants NS04053 (Epilepsy Research), NS05211 (Neurosurgery Training), NS09677 (Motoneuron Repetitive Firing Mechanisms) and NS09678 (Deafferentation) from the National Institute of Neurological Diseases and Stroke. Dr. O'Neal's present address is Division of Neurosurgery, University of Texas Medical Branch, Galveston, Tex. Dr. Westrum holds a joint appointment with Department of Biological Structure. 
origin of normal doublet activity and a candidate for the deafferented burst activity. Abnormal hyperactivity after deafferentation may be a function of changes in the membrane characteristics occurring at or near the denuded postsynaptic specializations.

\section{INTRODUCTION}

Claude Bernard, in 1872, proposed the general concept that tissue excitability may be increased by isolation from the controlling nervous input (3). Cannon and Rosenblueth further elaborated this concept and formulated a theory of "deneryation supersensitivity" applicable to all excitable structures from smooth muscle to the central nervous system (8). In the ensuing 30 years, a number of students have investigated partial deafferentation of the central nervous system as reflected in abnormalities of motoneuron output and muscular response. Extracellular recordings from deafferented spinal cord demonstrated not only alterations of spontaneous neuronal activity, but also abnormal evoked responses (17). In addition extracellular recordings in the spinal trigeminal nucleus have shown similar altered firing patterns (1).

Deafferentation has produced comparable abnormalities in spontaneous neuronal activity patterns in the lateral cuneate nucleus (LCN) of cats. Our previous papers $(14,19)$ have described in detail the neurophysiological and fine structural features of this form of partial deafferentation. In the present paper, we shall correlate these individual observations to establish a background for discussion of the possible mechanisms of neuronal hyperactivity and repetitive firing.

\section{MATERIALS AND METHODS}

This paper utilizes a portion of the anatomical and physiological data reported separately in two other papers $(14,19)$. Adult cats $(2-5 \mathrm{~kg}$ ) with survival times of 24 and $45 \mathrm{hr}, 6,10$ and 16 days, have been selected to correlate the structural and functional changes observed after $\mathrm{C}_{1}-\mathrm{T}_{7}$ dorsal rhizotomy. Two animals for each survival time were used, one for physiological and the other for anatomical studies. The surgical techniques employed to accomplish the deafferentation have been described in detail $(14,19)$. They are the same for each member of a pair of animals except that the $\mathrm{T}_{1}$ root was preserved in the animals for physiological recording to allow characterization of units retaining their peripheral receptive field in the deafferented nucleus. The methods of tissue fixation and handling for electron microscopy, as well as the techniques for physiological recording, have been described in detail in our previous papers $(14,19)$.

The physiological data analysis techniques employed in this paper are basically those described by Calvin (5). The definition of a burst is the 
property of a computer program which scans the list of interspike intervals stored on computer tape after sampling from the original data recordings. This program looks for interspike intervals longer than $30 \mathrm{msec}$; upon finding such a long interval, it examines the very next interval to see if it is shorter than $10 \mathrm{msec}$. If both criteria are satisfied in succession, the short interval is considered to denote the beginning of a doublet or burst. The "long" and "short" interval criteria may be varied by the computer operator; while 30 and $10 \mathrm{msec}$ were common values, 15 and $5 \mathrm{msec}$ were also used for one illustration. This burst detection scheme is utilized by the programs which make rasters and histograms. This scheme is not infallible; indeed, one can detect occasional examples of the inclusion of nonbursts in the burst rasters in this paper and the previous paper (14). Sampling time bins were $0.2 \mathrm{msec}$, in duration.

\section{RESULTS}

A detailed description of the normal fine structural architecture and physiological characteristics of the cat lateral cuneate nucleus (LCN) was included in the previous papers $(14,19)$. As a basis for later discussion, a description of the normal repetitive firing mechanisms is introduced here.

One form of normal spontaneous activity in $L C N$ includes neurons with a very high firing rate capability, appearing as doublets and, occasionally, triplets. Calvin (6) distinguished three models for multiple spike production which are germane to analysis of repetitive firing in LCN. Most commonly, a large depolarizing wave will evoke multiple spikes by converting the depolarization magnitude into firing frequency. The spikes following the initial spike arise out of the interaction of a sustained synaptic depolarization with an oscillator mechanism. These oscillator-produced spikes bear no relationship to the timing of the various inputs. As the depolarizing current increases, the interspike interval decreases. Characteristic of this mechanism, then, is a fluctuating depolarizing drive reflected in a variable interspike interval. The brevity of the doublet activity and its relative invariability in LCN make it doubtful that a depolarization-to-frequency model can adequately account for the phenomenon.

A second model accounts for a repeated action potential response via an EPSP-produced spike; in this model, the EPSP from a single input may be almost large enough to fire the cell. Here, the timing of the input and output spikes is correlated. This model may be applicable to the Clarke's column cells where single IA fibers may produce EPSP in the 2-5 mv range (11) and, by analogy, to the LCN cell where presynaptic profiles are large and probably establish multiple contacts with postsynaptic 
elements. While such a large EPSP might produce the first spike, it would be unlikely that a second subsequent EPSP could produce the second spike and maintain the precise timing between the first and second spike of the doublet. This would tend to rule out sequential EPSP from two different afferent fibers, as they could not be expected to retain such synchrony. It is also unlikely that multiple contacts from a single bifurcating afferent fiber would produce two successive EPSP separated by an interval of 1-1.6 msec (which was the olsserved interspike interval separation in I.CN).

The third model for repetitive firing is found in the depolarizing afterpotential theory $(6,13)^{\circ}$. The depolarizing aftermath of the initial spike may rise through threshold and cause an extra spike, even if the original depolarizing influence has dissipated. The second spike is correlated with the peak of the delayed depolarization (DD) from the first spike. If the DD peak occurs within 2 msec, one would expect the extra spike to occur before 2 msec. Inspection of Fig. 1 of this paper and Fig. 1 of the preceding paper (14) demonstrates the second spike occurring within $2 \mathrm{msec}$ of the first spike. The few existing intracellular records from the LCN are not of sufficient quality to demonstrate this depolarizing afterpotential if it does exist $(9,21)$. It is interesting, however, that the homologous Clarke's column cells invariably present a delayed depolarization, peaking about 2 msec following the initiation of the spike $(10,11)$. Thus, for the present, as in spinal motoneuron (7) and hippocampal pyramidal cell (13), it seems plausible that the spike-evoked spikes mechanism is sometimes utilized by the I.CN cell under normal circumstances. Peripheral needle electrode stimulation of LCN receptive fields yields a train of spikes from cells in the normal nucleus. This artificial, synchronized input probably acts through a potent synaptic effect and oscilator-produced spikes (16).

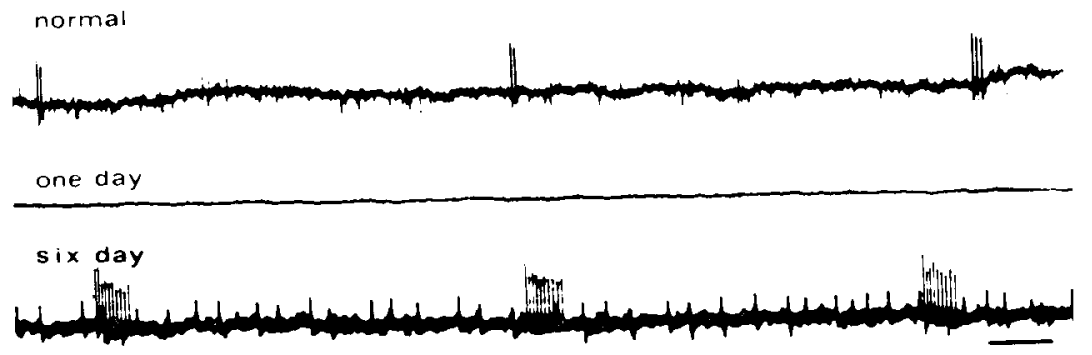

Fig. 1. Spontancous activity in LCN. Upper trace, normal cat; middle trace, 24 hr after ipsilateral dorsal rhizotomies of $C_{1}-T_{7}$, sparing $T_{1}$. Note the absence of unit activity: Lower trace, 6 days after ipsilateral dorsal rhizotomies of $C_{1}-T_{7}$ sparing $\mathrm{T}_{1}$. Note the hyperactivity and burst firing patterns. Calibration: $10 \mathrm{msec}$. Spikes are about $200 \mu \mathrm{v}$. Gain in 1-day trace is half of others. 
Deafferented Animals. After dorsal rhizotomies, two distinct phases of abnormal neuronal activity are associated temporally with morphological alterations in LCN (Table 1). The earliest physiological change consists of almost complete absence of spontaneous electrical activity in I.CN by $2+$ hr after $\mathrm{C}_{1}-\mathrm{T}_{\gamma}$ dorsal rhizotomies (Fig. 1). Irregular, single spike activity can be evoked by tetanic stimulation of the cerebellum. At this time, the primary morphological alteration is diminution in numbers of synaptic

TABLE: 1

CORKLLATION OF P'hysiological WTth MORphological Chavies in LCCN AFter Dorsal Rhizómomits

\begin{tabular}{|c|c|c|}
\hline Survival time & Physiological changes ${ }^{\prime}$ & Anatomical changes \\
\hline $24 \mathrm{hr}$ & $\begin{array}{l}\text { SA: "silent" nucleus } \\
\text { AD: augmentation of solitary } \\
\text { spikes }\end{array}$ & $\begin{array}{l}\text { Decrease in numbers of } \\
\text { synaptic vesicles }\end{array}$ \\
\hline $45 \mathrm{hr}$ & $\begin{array}{l}\text { SA: abnormal firing patterns, } \\
\text { higher than normal } \\
\text { rates. } \\
\text { AD: augmentation of irregular } \\
\text { firing, and burst dis- } \\
\text { charges }\end{array}$ & $\begin{array}{l}\text { Degenerating boutons } \\
\text { mainly renaining at- } \\
\text { tached to postsynaptic } \\
\text { sites }\end{array}$ \\
\hline 6 days & $\begin{array}{l}\text { S.l: burst firing and ir- } \\
\text { regular high frequency } \\
\text { solitary spikes; more } \\
\text { units firing per re- } \\
\text { cording site }\end{array}$ & $\begin{array}{l}\text { Removal of some degener- } \\
\text { ated boutons; unit mem- } \\
\text { brane structure exists } \\
\text { over denuded postsynap- } \\
\text { tic specializations; mod- } \\
\text { crate increase in extra- } \\
\text { cellular space. }\end{array}$ \\
\hline 10 days & $\begin{array}{l}\text { SA: burst firing and irregular } \\
\text { high frequency spikes; } \\
\text { instability of firing } \\
\text { patterns } \\
\text { AD: augmentation of burst } \\
\text { duration and tonic } \\
\text { single spike activity }\end{array}$ & $\begin{array}{l}\text { Most degenerated boutons } \\
\text { removed; unit membrane } \\
\text { structures still persist } \\
\text { over postsynaptic special- } \\
\text { izations; loss of dendritic } \\
\text { spines; marked increase } \\
\text { in extracellular space. }\end{array}$ \\
\hline 16 days and later & $\begin{array}{c}\text { SA : burst firing and irregular } \\
\text { high frequency spikes } \\
\text { AD: same as } 10 \text { days }\end{array}$ & $\begin{array}{l}\text { Disappearance of most unit } \\
\text { membrane structures at } \\
\text { denuded postsynaptic } \\
\text { specializations; additional } \\
\text { loss of dendritic spines; } \\
\text { extracellular space nearly } \\
\text { normal. }\end{array}$ \\
\hline
\end{tabular}

a SA, spontaneous activity; AD, antidromic activity elicited by tetanic cerebellar stimulation. 


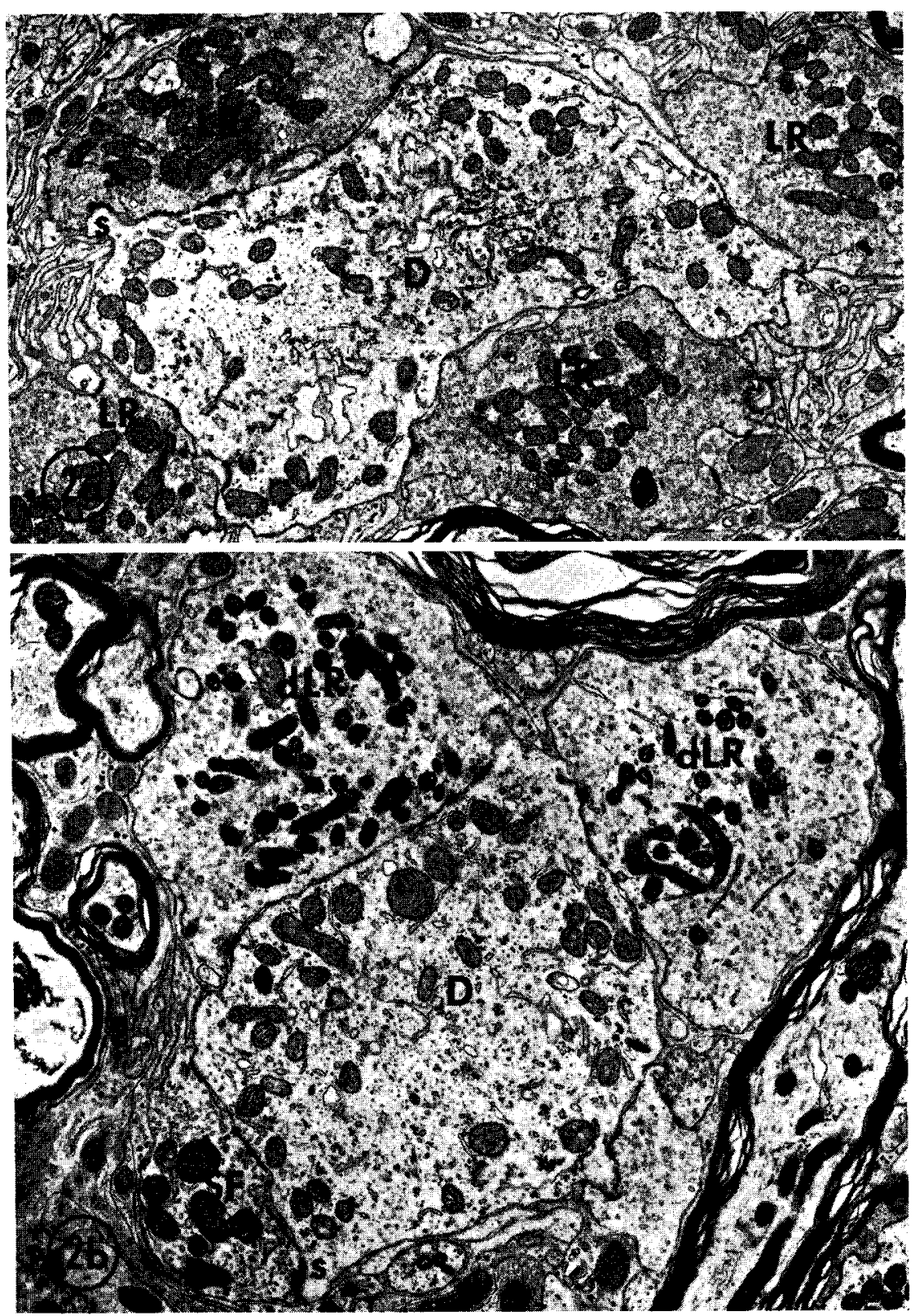

FIG. 2. (a) A preparation from $\mathrm{LCN}$ of a normal cat showing the frequently occurring large boutons with round vesicles (LR) contacting a medium-sized dendrite (D) both directly and via a spine (s). $\times 11,700$. (b) Twenty-four hours survival Moderate vesicle depletion in swollen LR boutons (dLR) and an unaltered SF bouton contacts a dendrite (D) and spine (s). $\times 11,700$. 
vesicles in the large presynaptic profiles with round vesicles (LR boutons) (Fig. 2). This diminution ranges from a slight decrease in numbers of synaptic vesicles to almost total depletion. In some boutons where vesicle loss is incomplete, the remaining vesicles are aggregated and pleomorphic. The majority of LR profiles in LCN seem to show these changes.

The second phase of abnormal neuronal activity develops between $45 \mathrm{hr}$ and 10 days after dorsal rhizotomies. It is characterized initially by an augmented response to tetanic cerebellar stimulation (2-day survival) which consists of repetitive, single spikes interspersed with burst firing. This evoked form of hyperactivity is succeeded by a similar, but spontaneously occurring, form of rhythmically recurrent bursting by 6 days after deafferentation. As previously described (14), burst recurrence rates tend to vary from unit to unit $(6-12 / \mathrm{sec})$ but are remarkably regular for each individlual unit. Cessation of each burst is typically abrupt with only a slight decline in frecuency prior to termination (Fig. 1)

The structural changes which occur between 2 and 6 clays aiter dorsal rhizotomies are characterized by a sequential progression of degeneration of essentially all I.R boutons from electron-lucent to electron-dense forms (Fig. 3). A few denuded postsynaptic specializations (as in Fig. 5) are identifiable by 6 days; however, many of the degenerating boutons remain attached to postsynaptic elements at this time. The small presynaptic pro-

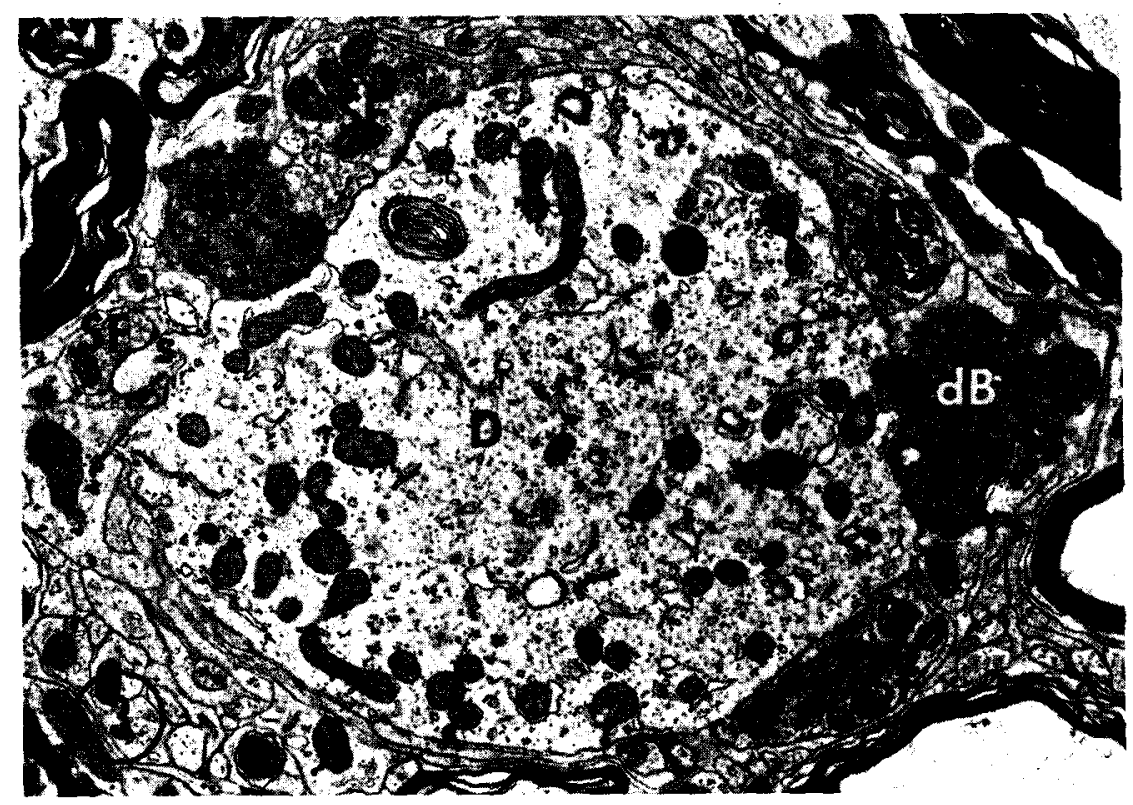

Fig. 3. Six days survival. Dense degenerated boutons (dB) are attached to a dendrite (I)). SF boutons contact the dendrite and a spine (s). $\times 12,150$. 

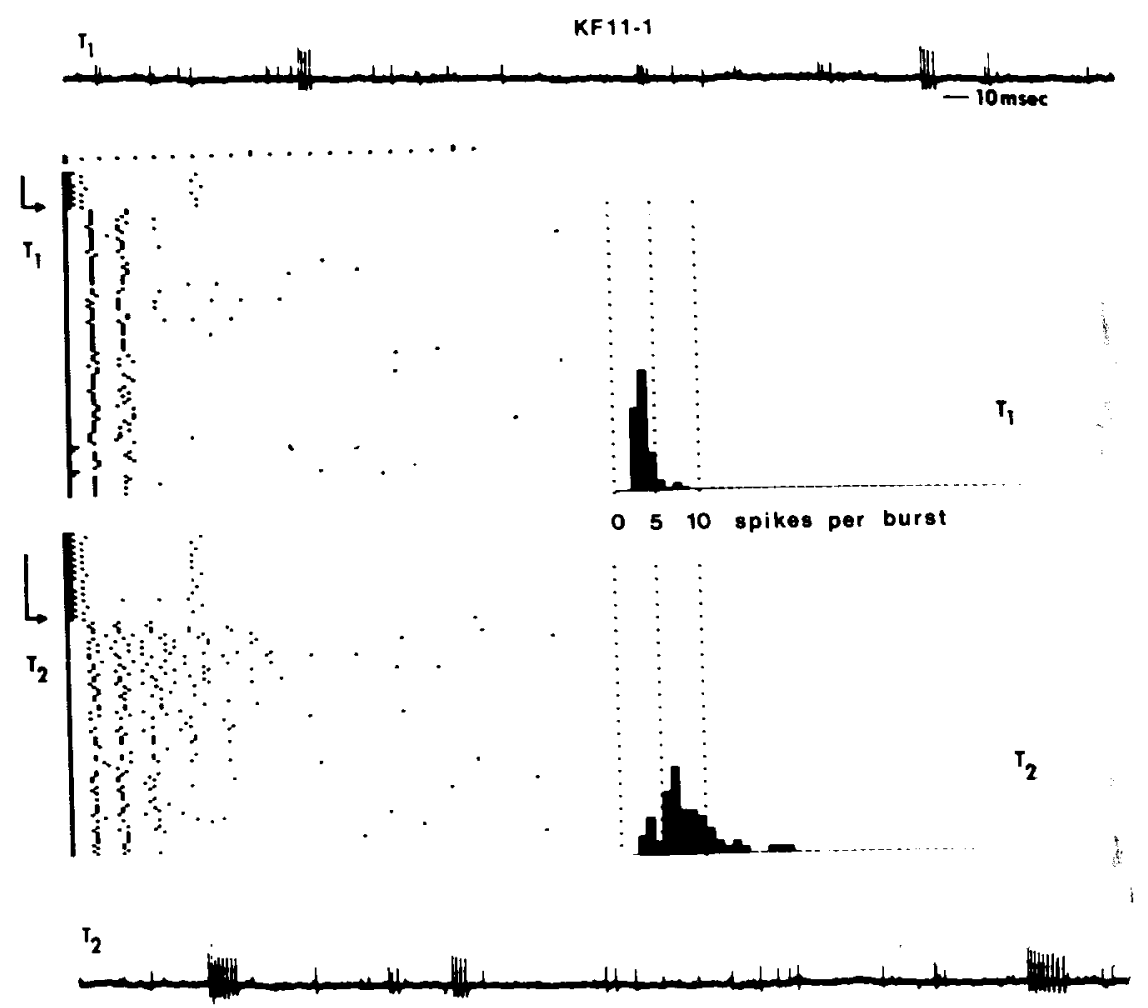

Fig. 4. Augmented burst response in LCN. Oscilloscope trace $T_{1}$ shows a burst (3-4 spikes/burst) recurring at $6 / \mathrm{sec}$ following tetanic cerebellar stimulation. Raster $\mathrm{T}_{1}$ corresponds to the oscilloscope trace. Note the end of tetanic stimulation at the toy of the raster. At upper right is the spikes per burst histogram for the $T_{1}$ activity. Oscilloscope trace $\mathrm{T}_{2}$, immediately following second application of tetanic stimulus, shows a burst (4-9 spikes/burst) recurring at $7 / \mathrm{sec}$. Raster $T_{2}$ corresponds to the $T_{2}$ oscilloscope trace. Note the end of tetanic stimulation at the top of the raster and the following immediate increase in spikes/burst with no alteration of the interspike interval. The $\mathrm{T}_{22}$ histogram demonstrates the corresponding spike/burst mode. The $10-$ msec calibration mark applies to both oscilloscope traces. The vertical rows of dots on the histograms mark 0,5 , and 10 spikes/burst. Spike amplitude $350 \mu v$.

files with flattened synaptic vesicles (SF boutons) in LCN apparently do. not undergo any morphologic alterations.

By 10 days after dorsal rhizotomies the spontaneous activity pattern in LCN, which was rather stereotyped for any given unit at earlier survivals, now manifests unstable firing patterns. The previously characteristic high frequency bursts alternate with shorter bursts and a tonic pattern of solitary spikes. This spontaneous cycling activity seems to be unrelated to extraneous factors such as blood pressure or respiration. In addition the 


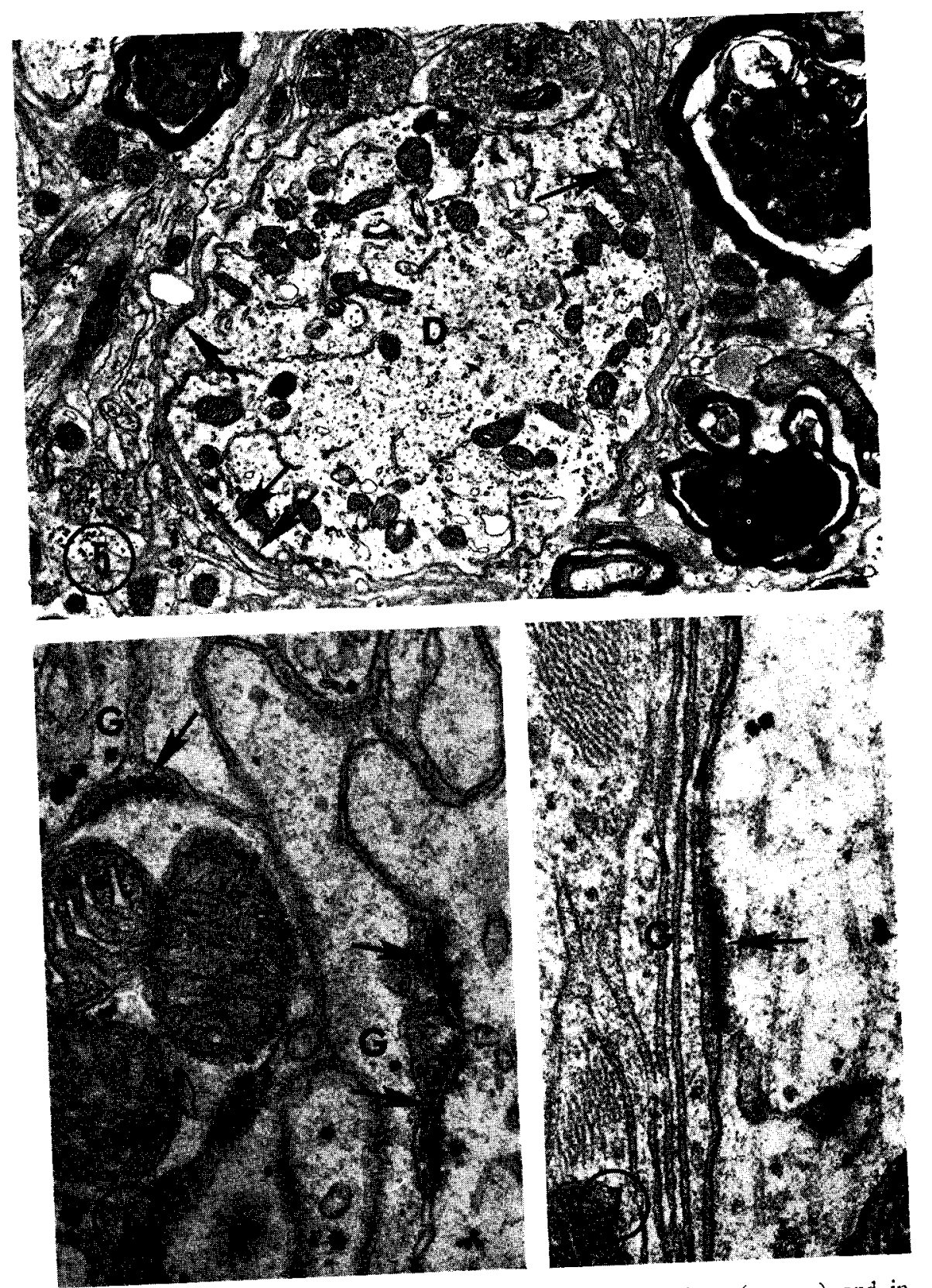

FIG. 5. Ten days survival. Denuded postsynaptic specializations (arrows) and increased extracellular space (asterisks) are indicated. Unaltered $S F$ boutons persist. $\times 12,150$. Fig. 6. Ten days survival. Denuded plial profiles $(G) . \times 44,100$. structures (arrows) and there are apposed glial profles (G). $\times 44,100$. 
angmented response to tetanic cerebellar stimulation first discernable in the $45 \mathrm{hr}$ survival is still present (Fig. 4). Associated with these physiological alterations is an increase in the numbers of denuded postsynaptic specializations previously apposed by degenerating LR profiles (Fig. 5). After removal of these LIR boutons the extracellular space adjacent to the denuded postsynaptic specializations is larger than normal and, at least transiently, oftet measures up to about $0.2 \mu \mathrm{m}$. 'This localized increase in extracellular space is most pronounced around medium to large dendrites 10 days after deafferentation (Fig. 5).

Concomitant with (glial) removal of clegenerated LR profiles and associated temporally with the physiological changes of the 6-and 10-day survivals, two further anatomical alterations occur. A unit membrane-like structure (Fig. 6 ) is visible in the extracellular space directly over denuded postsynaptic specializations. On its other side, i.e., away from the postsylaptic specialization, this structure may be apposed by a variety of elements which include glia, dendrites, axons or SF boutons (Fig. 6). Secondly, the number of dendritic spines seems to decrease. Those spines that remain are contacted by SF boutons as in Fig. 3.

I'hysiologically, the abnormal neuronal firing patterns summarized above seem firmly established by 10 days after dorsal rhizotomies. No additional alterations in spike activity could be clemonstrated in animals with longer survivals. It must be emphasized that those units in $1 . C N$ which responded to peripheral stimulation ( $T_{1}$ root was spared in those animals utilized for physiological study) never manifested the abnormalities seen in units which had lost their afferent drive (14). They retained all the features of the normal response to cerebellar and peripheral stimulation (Fig. 8). The morphological picture is also essentially stable by this time. It consists of those features described for 10 days survival with the addition of two new alterations: an apparent loss of many of the unit membrane-like structures in the extracellular space over denuded specializations; and an apparent decrease in the size of the enlarged extracellular space seen adjacent to denuded specializations in the 10-day survival (Fig. 7). Both alterations take place betwcen 10 and 16 days after dorsal rhizotomy.

\section{DISCLSSION}

The two clear-cut phases in the development of neuronal firing changes seem to be correlated with the concomitant fine structural alterations. The earliest physiological change is relative electrical silence within LCN which

Fig. 7. Sixteen days survival. A denuded postsynaptic specialization (arrow) is apposed by glial lamellae $(G)$ and appears to have lost the unit membrane structure. $\times 46,800$. 

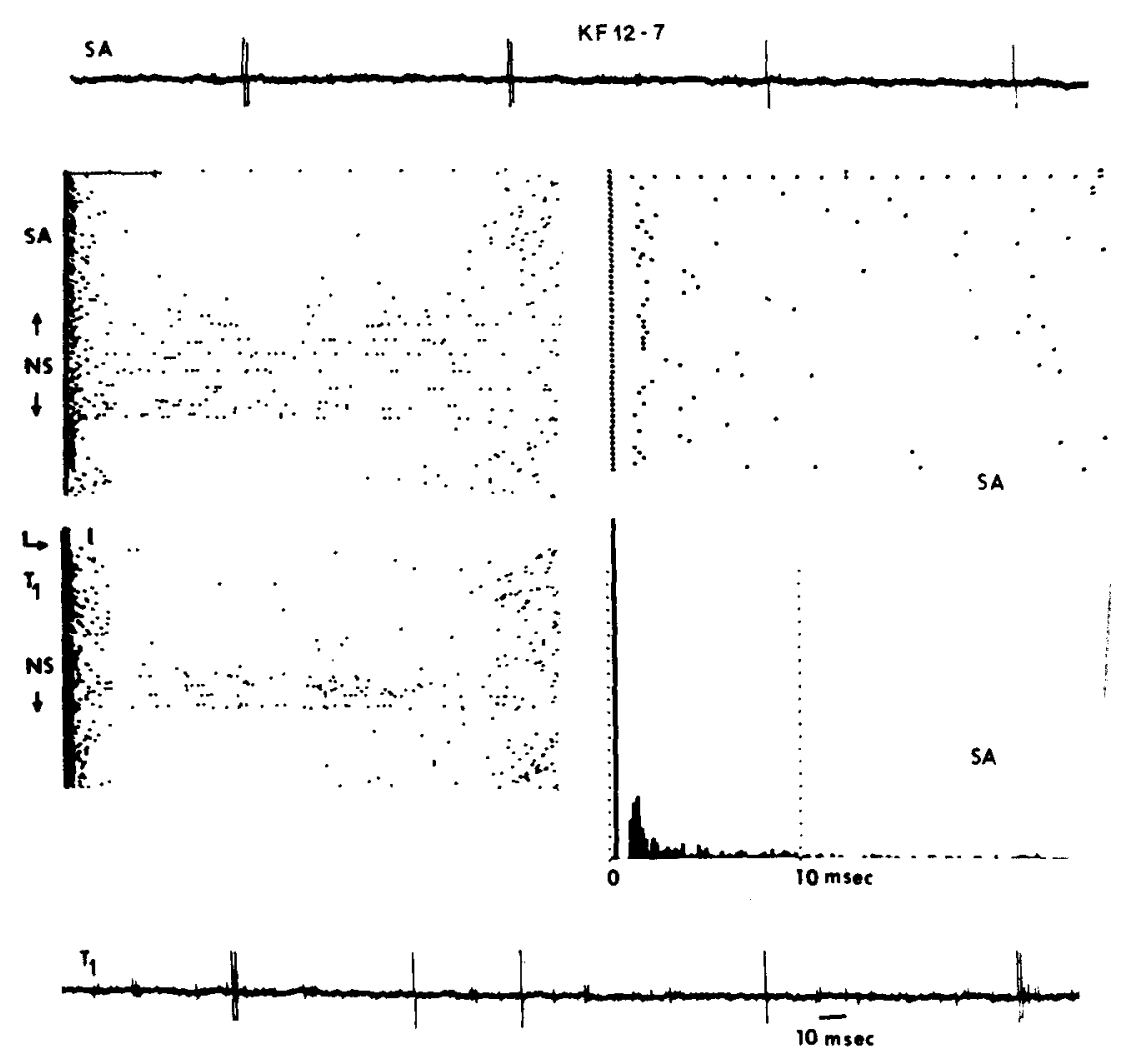

Fig. 8. Normal spontaneous and evoked activity of unit with intact peripheral receptive field. Oscilloscope trace KF12-7 illustrates a $400 \mu \mathrm{v}$ unit with doublet firing node recurring at $18 / \mathrm{sec}$. The combined spontaneous activity-natural stimulation raster (SA, NS) shows the prestimulation doublet and then the increase of activity secondary to foot-pad pressure. The time scale at the top of the raster shows a dot every $1 \mathrm{msec}$ for $20 \mathrm{msec}$, then one dot for each subsequent $10 \mathrm{msec}$. The $\mathrm{T}_{1}$, NS raster (combination of activity immediately following tetanic cerebellar stimulation and peripherally evoked activity during this period) has the same time scale. At the top left of the raster is the end of the tetanic stimulation period. Note the failure of tetanic stimulation to alter firing frequency. There is a spread of activity midraster subsequent to natural stimulation. The SA raster at upper right demonstrates the spontaneous activity before tetanic cercbellar stimulation at an expanded time scale. The time scale at top marks each $1 \mathrm{msec}$. The SA histogram shows the probability density of spikes. The oscilloscope trace $T_{1}$ (immediately following tetanic cerebellar stimulation) shows a $400 \mu \mathrm{v}$ unit recurring at $22 / \mathrm{sec}$. The $10-\mathrm{msec}$ calibration applies to both kymograph traces. Unit $\mathrm{KF} 12-7$ was a cutaneous unit (C-CCT) responding to foot pad pressure.

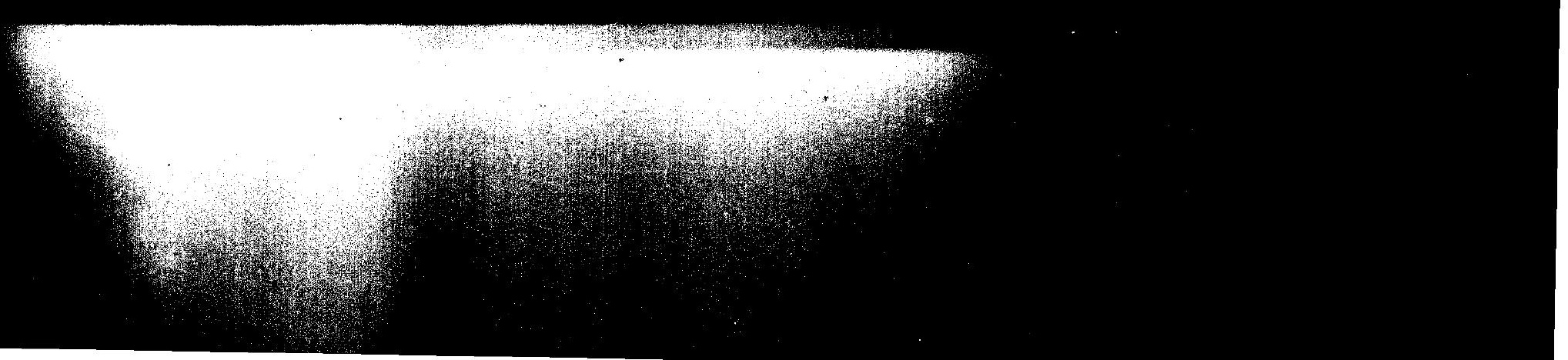


is presumably a manifestation of the loss of afferent drive. Previous investigations in both the peripheral and central nervous systems have suggested an association of transmission failure with depletion of synaptic vesicles. At the neuromuscular junction, Birks, Katz and Miledi found synaptic vesicle reduction at a time when failure of transmission and loss of spontaneots end plate activity were seen (4). Hunt and Nelson also demonstrated a similar association early in the course of degeneration in denervated frog sympathetic ganglion (12). In the CNS an identical association has been documented for transmission failure in the cat lateral geniculate nucleus $(20,22)$. The decreased activity in LCN, while not specifically indicative of synaptic transmission failure, does correspond to a marked reduction of synaptic vesicles in degenerating I.R boutons. Transmission failure may be the basis for the decreased neuronal activity, although it is unlikely that axonal conduction failure has occurred at this time (18).

The second phase of the reaction to deafferentation commences between 2 and 10 days after dorsal rhizotomies. Marked alterations of resting activity make their appearance during this period. These are usually characterized by the development of spontaneous hyperactivity in the form of burst firing, and unstable firing patterns which involve alternating or cycling modes of firing. During this period, electron microscopy shows degeneration of essentially the entire population of L.R boutons and the progressive glial removal of these elements from their postsynaptic specializations. By 10 days, when unstable firing patterns are observed, the most characteristic anatomical alteration is denudation of postsynaptic specializations accompanied by an increase in the extracellular space near these sites and an apparent reduction in the number of dendritic spines. The denuded postsynaptic specializations are apposed by a unit membrane-like structure during this period. Similarly in the trigeminal nucleus following deafferentation, numerous denuded postsynaptic specializations were seen at a time concurrent with neuronal hyperactivity (24). The trigeminal study did not describe spine loss or increased extracellular space. The observations common to these two regions supports the conclusion that denuded postsynaptic specializations may form at least one anatomical concomitant to neuronal hyperactivity after deafferentation.

The increased postsynaptic neuronal activity which follows dorsal rhizotomies may be due to increased synaptic currents (perhaps due to the proliferation of chemically sensitive sites) or to alteration in the repetitive firing mechanisms of the postsynaptic cell (perhaps due to changes in electrical excitability). Certainly, a definite correlation between the observed morphological alterations and resting activity changes awaits good intracellular records from such a deafferented population of cells.

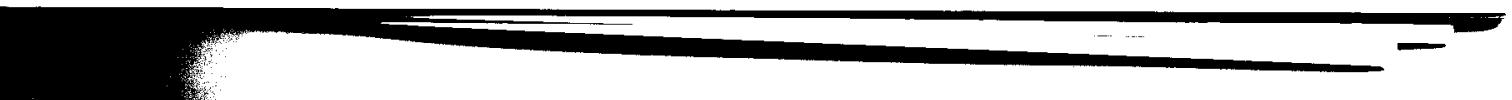



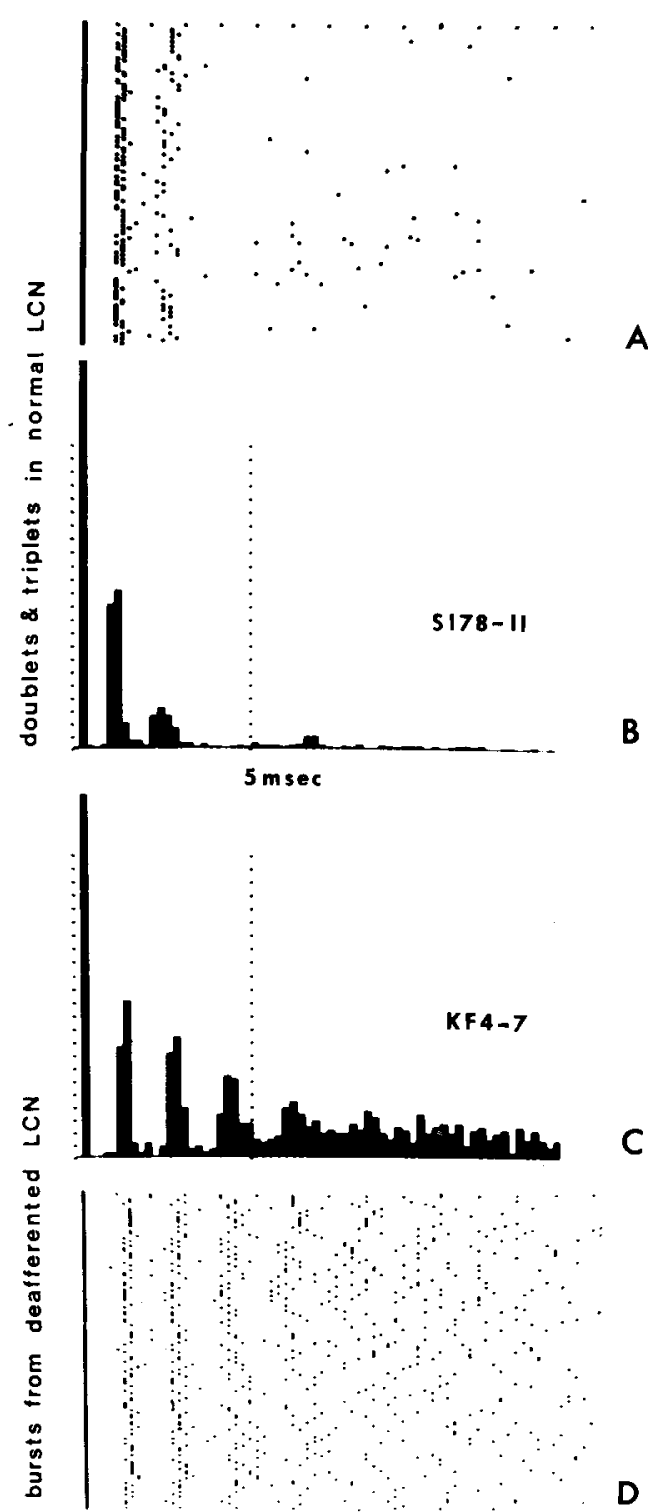

Fig. 9. Normal doublet and deafferented burst activity compared by raster displays. (A) Raster of normal LCN neuron which fired in doublet firing pattern. Computer aligns first spikes beneath one another producing solid line at left. (B) Probability density for (A), showing probability of finding a spike at various times after an initial spike (see methods). (C and D) Probability density and raster for bursts from a 10 day deafferented neuron from a different animal. Note the similarity in the initial interspike intervals of the burst when compared to the normal doublet firing patterns. 
There is suggestive extracellular clata, however, of nuttiple types of repetitive firing mechanisms operating in this nucleus. With the population of the I.R boutons degenerating, the EPSP-produced spike response presumably would disappear. Therefore, by exclusion, either the spike-evoked spikes mechanism or the oscillator-produced spikes model might be expected to operate on the deafferented postsynaptic membrane and give an increased number of spikes per burst. The source of the depolarization required to initiate spikes via either mechanism is, however, probematical. The remaining SF boutons could modify the membrane potential. Pacemaker mechanisms (23) might develop.

The burst firing in deafferented I.CN neurons may not be due exclusively to one mechanism, just as the doublet firing patterns in normal motoneurons are produced by a mixture of the spike-evoked spike mechanism and the oscillator mechanism ( 7 ). The labile firing patterns seen 10 days after dorsal rhizotomy suggest such a mixture: while the firing pattern was indeed bursting some of the time, it gradually changed and then stopped bursting altogether (Fig. 6 in Ref. 14). Such behavior is suggestive of variations in the synaptic drive and hence some involvement of the graded depolarization-to-frequency oscillator mechanism for producing spikes.

The best evidence for the spike-evoked spike hypothesis in the present data comes from regularly bursting units (as depicted in Figs. 1 and 8). The doublet firing patterns seen in the normal I.CN neurons suggest the spike-evoked spike mechanism. If the mechanisms which limit the second spike from evoking a third spike, the third from evoking a fourth, etc. are altered, one might expect a burst instead of a doublet. Figure $9 \mathrm{com}$ pares the doublet firing pattern from a normal animal with the burst patterns from an animal 10 days after dorsal rhizotomy. It is evident that the initial interspike intervals are similar. The occasional third spike (triplets) in the normal neuron suggests that the spike-evoked spikes mechanism is not limited to two spikes as tightly as it seems to be in cat spinal motoneurons $(7)$.

The problem of burst limitation remains to be discussed. The regularly recurring, high frequency bursts have an abrupt cessation with terminal firing rates of $500 / \mathrm{sec}$. Comparison of this latter mode of burst termination with that of injured units (decremental amplitude, increasing interspike interval and spike duration) suggests a physiological mechanism for burst cessation. The residual SF bouton population might be related to this termina-

Note also the frequent triplets in the normal neuron's raster (A). This similarity suggests that deafferentation may alter a normal repetitive firing mechanism (such as the spike-evoked spikes model discussed in the text).

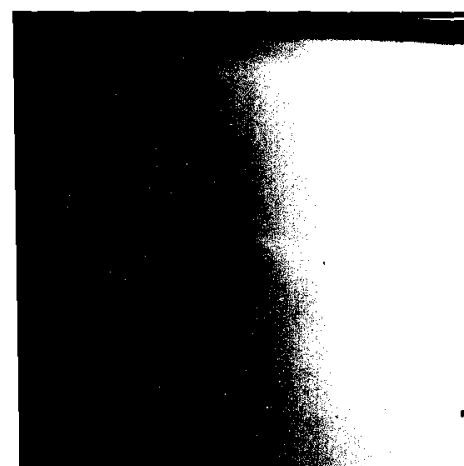


tion process. Of course, cessation of the burst need not involve a synaptic relationship. It may simply be a feature of the postsynaptic membrane: the delayed depolarization may be labile and become smaller with the successive spikes, or an afterhyperpolarization may build up after several spikes $(7,16)$.

Spikes are thought to be initiated near the beginning of the axon of typical central nervous system neurons. Then they antidromically invade the soma-dendritic region while the axon spike is propagating ortho(lromically. It is this normal antidromic invasion which probably gives rise to both the afterhyperpolarization and the delayed depolarization ( 7$)$. The delayed depolarization apparently involves dendritic invasion. The afterhyperpolarization involves both somatic and dendritic invasion. The afterhyperpolarization would seem essential for the oscillator mechanism, e.g., the extent of the antidromic invasion might control the oscillator's firing rate. Thus, both oscillator-produced spike and spike-evoked spike theories involve the electrical properties of the soma-dendritic membrane.

The present investigation provides no direct evidence to support an increased synaptic current developing secondary to the spread of chemically sensitive sites, as seen at the neuromuscular junction (2) and the postganglionic parasympathetic cell (15). The increased extracellular space which seems to develop concomitant with removal of degenerating I R boutons may act as a reservoir for various chemical substances whose net effect on the postsynaptic element cotld be excitation. Furthermore, should transmitter-like substance occur in this space, the mechanisms of their removal may be inadequate, with the consequent possibility of prolonged transmitter action. However, the observed increase in extracellular space at 10 days seems to be a transient phenomenon. Since the alterations in resting activity are persistent beyond 10 days, it is difficult to attribute the observed neuronal firing abnormalities solely to this observation.

The spontaneous high-frequency bursts seen after dorsal rhizotomies also can be triggered by a single stimulus to the cerebellar cortex (Fig. 2 in Ref. 14). These triggered bursts retain the characteristic interspike intervals, again suggesting that spike-evoked spike model might be operative. In addition, tetanic cerebellar stimulation leads to the augmentation of burst length (Fig. 4) with little or no change in the initial interspike intervals.

In the normal LCN, tetanic cerebellar stimulation occasionally leads to a suppression of spontaneous activity. Presumably, the apparent inhibitory influcnce is mediated by the SF boutons, since all LR boutons seem to come from dorsal root fibers. In the deafferented LCN, tetanic cerebellar stimulation should activate this inhibitory mechanism; yet the postsynaptic response is augmentation of bursting activity. This implies that the post- 
synaptic membrane is the locus of the repetitive firing which characterizes the activity in I.CN after dorsal rhizotomies. The fact that tetanic cerebellar stimulation 24 hr alter dorsal rhizotomies produced irregular, solitary spikes at a rate faster than the resting level, at a time when no apparent postsynaptic changes with the electron nicroscope are seen, may suggest early functional postsynaptic membrane changes. However, since a single antidromic stimulus occasionally yields a monophasic spike following the antidromic spike by about $2.5 \mathrm{msec}$, it is also possible that these solitary spikes represent an orthodromic transsynaptic phenomenon (21). However, there is apparently no anatonical data to suggest such a direct cerebellar cortical projection to LCN.

\section{REFERINCES}

1. Axperson, I. S., R. G. Black, J. Abraham, and A. A. Ward, Jr. 1971. Neuronal hyperactivity in experimental trigeminal deafferentation. J. Neurosurg. 35 : $444-452$.

2. Axelsson, J., and S. A. Thesleff. 1959. Study of supersensitivity in denervated mammalian skeletal muscle. J. Fhysiol. $147: 178-193$.

3. BerNakd, C. 1872. "Lecons de pathologie experimentale (et lecons sur les proprietes de la moelle epiniere)." J. B. Bailliere et Fils, Paris.

4. BIRKS, R., B. KATZ, and R. Miledi. 1960. Physiological and structural changes at the amphibian myoneural junction, in the course of nerve degeneration. $J$. Physiol. 150: 145-168.

5. Calvis, IV. II. 1968. Evaluating membrane potential and spike patterns by experimenter-controlled computer displays. Exp. Ncurol. 21: 512-534.

6. Culvin, W. H., 1972. Synaptic potential summation and repetitive firing mechanisms: input-output theory for the recruitment of neurons into epileptic bursting firing patterns. Brain Res. 39 : 71-94.

7. Calvin, W. H., and P. C. Schwindt. 1972. Steps in production of motoneuron spikes during rhythmic firing. J. Neurophysiol. 35: 297-310.

8. Cannon, W. B., and A. Rosenbluter. 1949. "The Supersensitivity of Denervated Structures." MacMillan, New York.

9. Cooke, J. D., B. Larson, O. Oscarsson, and B. Sjölund. 1971. Organization of afferent connections to cuneocerebellar tract. E.rp. Brain Res. 13: 359-377.

10. Eide, E., L. Fedina, J. Jansen, A. Lundeberg, and L. Vyklickŕ. 1969. Properties of Clarke's column neurones. Acto Physiol. Scand. 77: 125-144.

11. Eide, E., L. Fedina, J. Jansen, A. LundberG, and L. Vyklický. 1969. Unitary components in the activation of Clarke's column neurons. Acta Physiol. Scand. $77: 145-158$.

12. Hunt, C. C., and P. G. Nerson, 1965. Structural and functional changes in the frog sympathetic ganglion following cutting of the presynaptic nerve fibers. J. Physiol. $177: 1-20$.

13. Kandel, E. R., and W. A. Spencer. 1961. Electrophysiology of hippocampal neurons II. After potential and repetitive firing. J. Neurophysiol. 24 : 243-259.

14. Kjerdef, T. D., and J. D. Loeser. 1973. Neuronal hyperactivity following deafferentation of the lateral cuneate nucleus. Exp. Neurol. 39: 70-85. 
15. Kuffler, S. W., M. J. Dennis, and A. J. Harris. 1971. The development of chemosensitivity in extrasynaptic areas of the neuronal surface after denervation of parasympathetic ganglion cells in the heart of the frog. Proc. Roy. Soc. lond. Ser. B 177 : 555-563.

16. Keno, M., and J. T. Miyahara. 1968. Factors responsible for multiple discharge of neurons in Clarke's columir. J. Neurophysiol. 31: 624-638.

17. Loeser, J. D., and A. A. WARd, JR. 1967. Some effects of deafferentation on neurons of the cat spinal cord. Arch Neurol. 17:629-636.

18. McDosard, W. I. 1972. The time course of conduction failure during degeneration of a central tract. Exp. Brain Res. $14: 550-556$.

19. ONeAr, J. T., and I. E. Westrum. 1972. The fine structural synaptic organization of the lateral cumeate nucleus. A study of sequential alterations in degeneration. Brain Res. (In press).

20. Preci-Sanvedra, J., O. I. Vaccarezza, T. A. Reader, and F. Pasqualini. 1970. Synaptic transmission in the degenerating latcral geniculate nucleus. An ultrastructural and electrophysiological study. Exp. Neurol. 26: 607-620.

21. Raush, J. 1969. "Electrophysiology of the External Cuneate Nucleus of Cat." Doctoral Dissertation, Lniversity of Washington, Seattle, Wash.

22. Vaccarezza, O. L., T. A. Reader, E. Pasqualini, and J. Pecci-Sanvedra. 1970. Temporal course of synaptic degeneration in the lateral geniculate nucleus. Its dependence on axonal stump length. Exp. Neurol. 28 : 277-285.

23. WAChtel, H., and W. A. Wis.son. 1971. Slow hyperpolarizing waves in bursting neurons are produced by a regenerative, nonreversible, current source. Fed. Proc. 30B : 711.

24. Westrum, L. E., and R. G. Black. 1971. Fine structural aspects of the symaptic organization of the spinal trigeminal nucleus (pars interpolaris) of the cat. Brain Res. 25 : 265-287. 